Nouvelles perspectives en sciences sociales

Revue internationale de systémique complexe et d'études relationnelles

\title{
Les villes comme agents : simulation des futurs possibles du système urbain européen
}

\section{Lena Sanders}

Volume 5, numéro 2, mai 2010

Sur le thème de la simulation

URI : https://id.erudit.org/iderudit/044081ar

DOI : https://doi.org/10.7202/044081ar

Aller au sommaire du numéro

\section{Éditeur(s)}

Prise de parole

ISSN

1712-8307 (imprimé)

1918-7475 (numérique)

Découvrir la revue

\section{Citer cet article}

Sanders, L. (2010). Les villes comme agents : simulation des futurs possibles du système urbain européen. Nouvelles perspectives en sciences sociales, 5(2),

153-180. https://doi.org/10.7202/044081ar
Résumé de l'article

L'approche systémique pour modéliser la dynamique des systèmes de villes est ancienne. Le concept d'auto-organisation et le formalisme des équations différentielles ont donné lieu à de nombreuses applications dans les années 1980. La simulation agent ouvre de nouvelles perspectives dans ce champ. L'objectif de cet article est d'abord de discuter des registres et du niveau d'explication qui sont mobilisés pour rendre compte des différentiels de croissance des villes. Il s'agit ensuite de montrer l'intérêt d'une approche agent pour formaliser des hypothèses au niveau méso-géographique des villes. Après un bref état de l'art sur le concept de systèmes de villes et les modèles spatio-temporels associés, le modèle EuroSim formalisé avec un système multi-agents est présenté. Celui-ci permet de simuler l'évolution des villes européennes entre 1950 et 2050 en testant différents scénarios relatifs à l'ouverture des frontières vers l'immigration non européenne et à l'existence ou non de barrières économiques internes. 


\title{
Les villes comme agents : simulation des futurs possibles du système urbain européen
}

\author{
Lena Sanders \\ Laboratoire Géographie-cités, CNRS - Université Paris 1
}

\begin{abstract}
T a simulation multi-agents est une approche qui s'est rapide- 1 ment diffusée dans la communauté des modélisateurs en sciences sociales ${ }^{1}$. Cette approche est intrinsèquement dynamique, les interactions entre les entités constituant le moteur du changement, et elle implique la considération d'au moins deux niveaux, celui des entités micro qui sont en interaction, et celui des structures macro qui émergent de ces interactions. Ces propriétés expliquent l'intérêt de l'approche pour la modélisation en sciences sociales, champ où de nombreux phénomènes sont de nature multi-échelle. Le fait de pouvoir appréhender le niveau des individus est considéré comme un atout majeur dans de nombreuses applications, mais l'objectif de cet article est de
\end{abstract}

1 Joshua M. Epstein, Generative Social Science: Studies in Agent-Based Computational Modeling, Princeton, Princeton University Press, 2006; Timothy A. Kohler et George J. Gumerman (dir.), Dynamics in Human and Primate Societies. Agent-Based Modeling of Social and Spatial Processes, Oxford, Oxford University Press, 2000; Michael Batty, "Polynucleated Urban Landscapes ", Urban Studies, vol. 38, n 4, 2001, p. 635-655; Frédéric Amblard et Denis Phan, Modélisation et simulation multi-agents : applications pour les sciences de l'homme et de la société, Paris, Hermes-Lavoisier, 2006; Juval Portugali, "Toward a Cognitive Approach to Urban Dynamics », Environment and Planning B: Planning and Design, vol. 31, $\mathrm{n}^{\circ}$ 4, 2004, p. 589-613. 
montrer que cette approche est également innovante lorsqu'il s'agit de formaliser un jeu d'interactions entre des entités spatiales.

D'un point de vue thématique, l'article porte sur la croissance urbaine. Les villes concentrent une part de plus en plus importante de la population, au moins un habitant sur deux au niveau de la planète, sept sur dix en Europe en ce début du XXI ${ }^{e}$ siècle. Ces villes évoluent à des rythmes variés : certaines, très attractives, croissent et gagnent des places dans la hiérarchie urbaine (régionale, nationale ou internationale), alors que d'autres stagnent ou même déclinent, notamment en termes relatifs. Pourquoi ces différentiels de croissance? La question est importante tant du point de vue théorique - il s'agit d'expliquer ces différentiels en identifiant les facteurs et les processus sous-jacents, - que pratique - les aménageurs, les politiques, ont besoin de connaître les lieux de forte croissance ou de déclin pour anticiper services et infrastructures ou, au contraire, pour tenter de freiner une évolution considérée comme non désirable. La question de la différence de taille des villes a été largement étudiée et modélisée en géographie et en économie spatiale et la croissance des villes a donné lieu à de nombreux travaux tant théoriques qu'empiriques $^{2}$. Le champ est donc large et l'objectif de cet article est, d'une part, de discuter de la nature des explications qui sont mobilisées en fonction du niveau de modélisation choisi et, d'autre part, de montrer l'intérêt d'une approche agent pour formaliser des hypothèses faisant sens au niveau des villes. Après un bref état de l'art sur le concept et les modèles de systèmes de villes, on présentera le modèle EuroSim formalisé avec un système multi-agents (SMA). Celui-ci permet de simuler l'évolution des villes européennes dans une démarche combinant la reproduction des trajectoires passées (durant un demi-siècle) et une étude prospective sur un autre demi-siècle.

2 Denise Pumain, "Settlement Systems in the Evolution ", Geografiska Annaler, 82B, 2, 2000, p. 73-87. 


\section{Croissance urbaine : différents registres et niveaux d'ex- plication}

Le phénomène à expliquer peut s'exprimer suivant différents niveaux de généralité : quels facteurs expliquent qu'une ville $\mathrm{A}$ enregistre un taux de croissance soutenu? Pourquoi la ville $\mathrm{A}$ croît-elle alors que $\mathrm{B}$ décline? Pourquoi y a-t-il des différenciations interurbaines en matière de tailles de villes, en matière de croissance? Quels facteurs pourraient faire évoluer la hiérarchie urbaine vers une forme plus polycentrique? La première question réfere à une ville unique, la dernière appréhende l'ensemble du système urbain dans sa structure. Pour chacune d'entre elles, plusieurs registres explicatifs, correspondant à des niveaux géographiques différents, sont possibles. Avant de les aborder, il est utile d'expliciter comment est mesurée l'évolution d'une ville, sa propension à décliner ou à croître. L'indicateur le plus classique est le nombre d'habitants, qui est le plus souvent (mais pas toujours) corrélé avec l'importance de l'activité économique et le niveau fonctionnel de la ville. Cet indicateur dépend très directement de la délimitation spatiale de la ville qui va donc influer sur l'interprétation de son évolution démographique. Dans le cas de la France, par exemple, suivant que l'on considère la seule commune centre (principe administratif), l'unité urbaine (principe morphologique fondé sur un critère de continuité du bâti) ou l'aire urbaine (principe fonctionnel fondé sur la part des navetteurs travaillant dans l'unité urbaine), les résultats seront radicalement différents ${ }^{3}$. Dans une perspective interurbaine privilégiant une approche en termes de système de villes, la dernière définition est la plus pertinente. Sont alors comptabilisées comme habitants de la ville toutes les populations que celle-ci polarise, qu'elles soient localisées dans le centre ou en lointaine périphérie. Cette approche considère un espace urbain intégré, composé de lieux complémentaires qui vont contribuer ensemble à définir la position relative de la ville dans le système

3 Marianne Guerois et Fabien Paulus, "Commune centre, agglomération, aire urbaine : quelle pertinence pour l'étude des villes? ", Cybergeo, $\mathrm{n}^{\circ} 212$, 2002, p. $1-18$. 
des villes. De nombreux travaux ont pour objectif de constituer des bases de données harmonisées, en construisant des entités cohérentes d'un point de vue sémantique, pour permettre une comparaison internationale ${ }^{4}$.

Une fois les tendances à la croissance ou au déclin mesurées et constatées, il s'agit de les expliquer. Plusieurs candidats à l'explication co-existent.

- On peut considérer que la croissance ou le déclin d'une ville (d'un ensemble de villes) est le résultat des décisions individuelles des habitants en matière de migration et de choix résidentiel. Dans ce cas, le nombre d'habitants d'une ville est la résultante de l'ensemble des comportements individuels associés à cette ville et l'explication est à rechercher du côté des individus : pourquoi avoir choisi de rester dans la ville ou de la quitter? Pourquoi avoir choisi une ville donnée plutôt qu'une autre pour s'établir? Les modèles de microsimulation, par exemple, suivent cette logique $^{5}$. Le niveau de l'explication étant établi, différents principes peuvent être mobilisés. On peut ainsi distinguer les modèles qui cherchent à formaliser l'enchấnement causal conduisant un individu à migrer (l'individu A au chômage au temps $t$ dans la ville $X$ trouve un emploi au temps $\mathrm{t}+1$ dans la ville $\mathrm{Y}$ ), et les modèles s'appuyant sur une logique statistique (un individu de tel âge, de tel statut matrimonial et exerçant telle activité professionnelle, a une probabilité de tant de migrer). Les premiers reposent sur

$4 \quad$ Anne Bretagnolle, Villes et réseaux de transport, des interactions dans la longue durée (France, Europe, Etats-Unis), Habilitation à Diriger des Recherches, Université Paris 1, 2009.

5 Peter Wagner et Michael Wegener, "Urban Land Use, Transport and Environment Models. Experiences with an Integrated Microscopic Approach ", disP 170, 3, 2007, p. 45-56; Einar Holm, Urban Lindgren et Gunnar Malmberg, "Dynamic Microsimulation ", dans Stewart Fotheringham et Mikael Wegener (dir.), Spatial Models and GIS: New Potential and New Models, GISDATA 7, Taylor \& Francis, 2000, p. 143-165; Graham Clarke (dir.), Microsimulation for Urban and Regional Policy Analysis, London, Pion, 1996; Paul Waddell, "UrbanSim: Modeling Urban Development for Land Use, Transportation and Environmental Planning ", Journal of the American Planning Association, vol. 68, n 3, 2002, p. 297-314. 
des principes logiques référant à une théorie de l'action, les seconds sur des principes empiriques validés par les méthodes statistiques. Notons, par ailleurs, qu'il s'agit dans ces modèles individuels de distinguer deux étapes : celle concernant la décision de la migration et celle se rapportant au choix du lieu de destination.

- La croissance ou le déclin d'une ville peut aussi être vu comme le résultat d'une stratégie politique. L'exemple de Nîmes et de Montpellier est illustratif à cet égard. La figure 1 représente les poids respectifs de ces deux villes ${ }^{6}$ dans le système urbain français de 1831 à nos jours. Toutes deux déclinent régulièrement (en termes relatifs, pas en chiffres bruts) jusqu'au renversement que l'on observe dans les années 1960, où seule Montpellier regagne progressivement les places perdues dans la hiérarchie urbaine. Est-ce l'effet d'un marketing urbain dynamique? La municipalité de Montpellier « la surdouée » a, en effet, eu une démarche volontaire et active pour promouvoir la ville au niveau national et européen, afin d'attirer entreprises et populations et favoriser la croissance. Toujours à ce même niveau ville, la différence des deux trajectoires peut aussi simplement s'expliquer par des contextes socio-économiques différents, celui de Montpellier mieux à même de profiter de la diffusion des innovations dans le secteur des services, puis de la haute technologie, que Nîmes à la tradition plus industrielle ${ }^{7}$.

La première explication donne un poids prépondérant au rôle des acteurs, la seconde réfère à un modèle plus général de diffusion des innovations en fonction du contexte local.

$\overline{6}$ Dans cette figure, on considère les unités urbaines définies par l'Institut national de la statistique et des études économiques (INSEE) (privilégiant un principe de continuité du bâti) qui représentent des entités cohérentes pour une comparaison sur le temps long.

7 François Favory et al., "Sélection géographique, déterminisme et hasard ", dans Archaeomedes (collectif de direction), Des oppida aux métropoles, Paris, Anthropos, coll. «Villes ", 1998, p. 151-248. 


\section{Figure 1}

Evolution des positions relatives des agglomérations de Nîmes et Montpellier dans le système urbain français

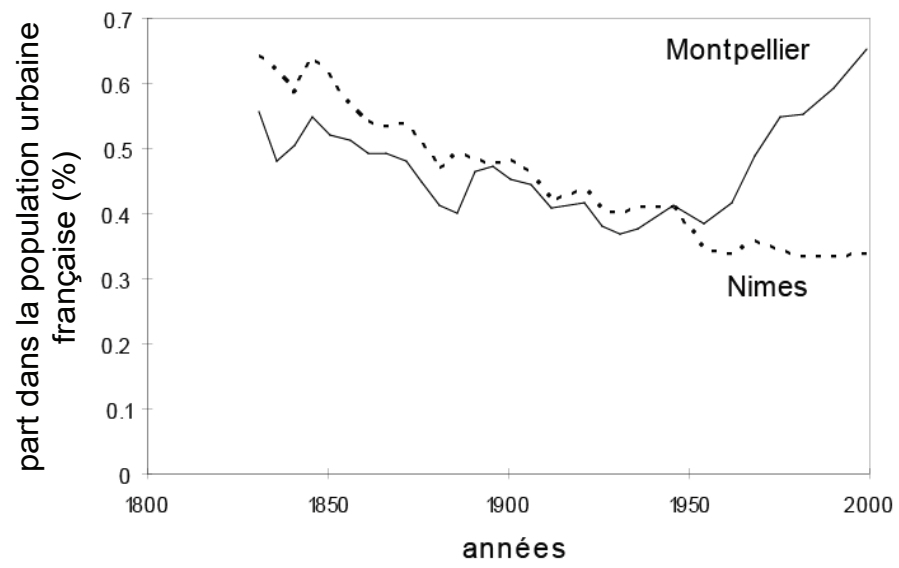

Source : François Favory et al., 1997

- Une troisième voie, enfin, consiste à appréhender les villes comme des entités collectives en interaction au sein d'un système auto-organisé, et à rechercher les explications de leur évolution dans ce fonctionnement systémique. D’une part, on peut évoquer le rôle des caractéristiques propres de la ville, sa taille (avec les économies d'agglomération sous-jacentes), son profil économique (et donc ses capacités d'innovation), ses ressources en termes d'environnement, de richesse, de gouvernance, ou encore son histoire (path dependency). D'autre part, on peut aussi considérer les villes comme les éléments d'un système et stipuler que l'évolution de chacune d'entre elles dépend de sa position relative dans ce système et de la forme et de l'intensité de ses interactions avec l'ensemble des autres villes. Ces deux dimensions explicatives sont évidemment liées : la forme des interactions engendre des différentiels entre les caractéristiques des villes, et ces différentiels induisent à leur tour des interactions d'intensités différentes. Le niveau élémentaire, celui où opèrent les interactions motrices, est 
alors celui des villes, et de ces interactions dépendent à la fois la propension à la croissance ou au déclin de chaque ville, et l'évolution de l'organisation hiérarchique et de la configuration spatiale du système des villes que l'on observe à un niveau supérieur. L'explication des différentiels de croissance est alors à rechercher dans la forme des interactions entre les villes et dans le lien entre ces interactions et la croissance.

Ces trois directions proposent ainsi des modes d'explication concurrents et se référant à des niveaux géographiques distincts. Elles correspondent à des positionnements épistémiques différents et ont chacune leurs implications ontologiques. La première relève de l'individualisme méthodologique et pour expliquer un fait urbain comme la croissance ou le déclin des villes, il s'agit d'étudier les aspirations et les préférences des individus. La ville correspond alors à un simple contexte dont les caractéristiques peuvent influer sur les choix individuels et, en tant qu'entité spatiale, elle représente un réceptacle, un espace support correspondant à un niveau d'agrégation auquel on comptabilise le nombre d'habitants. Cette perspective est ainsi bottom-up et s'inscrit dans une logique d'agrégation. La deuxième direction privilégie les acteurs urbains, les acteurs politiques et/ou économiques dont les décisions affectent l'organisation, l'image, l'attractivité de la ville prise comme un tout. Ces décisions peuvent s'articuler autour d'un projet commun et, dans ce sens, la ville peut être considérée comme un acteur collectif cherchant à valoriser sa position dans un contexte de compétition interurbaine ${ }^{8}$. La dernière direction adopte une perspective systémique et la ville est appréhendée comme un objet, une entité collective complexe, composée d'entités de nature différente, tant matérielles qu'immatérielles, humaines ou non (habitants, entreprises, réseaux techniques, infrastructures de transport, flux d'information etc.) dont les interactions contribuent à assurer l'identité, l'autonomie et la pérennité. Ces interactions internes peuvent

$8 \quad$ Patrick Le Gales, Le retour des villes européennes. Sociétés urbaines, mondialisation, gouvernement et gouvernance, Paris, Presses de Sciences Po, 2003. 
cependant être mises entre parenthèses quand on se concentre sur le rôle des interactions opérant à un niveau d'organisation supérieur, entre les entités villes. L'hypothèse est que les positions relatives des villes dans un système d'échange sont déterminantes pour expliquer les dynamiques différenciées de ces villes et l'évolution des caractéristiques spatiales et hiérarchiques du système de villes. Cette approche est également bottom-up, mais elle opère à d'autres niveaux géographiques puisqu'il s'agit des interactions entre les villes qui engendrent des transformations dans l'organisation du système des villes.

Chacun de ces candidats à l'explication privilégie un point de vue : la liberté de choix des individus pour la première, la marge de manœuvre des acteurs politiques pour la deuxième, l'effet des structures et de l'émergence pour la troisième. Ces différentes interprétations des différentiels de croissance entre les villes peuvent donner lieu à différents modèles Dans le premier cas, les entités élémentaires sont les individus, alors que les deux autres appréhendent directement le niveau des villes, l'une dans une perspective d'acteur collectif et référant à un projet, l'autre dans une perspective de système auto-organisé sans finalité a priori. Dans la suite de l'article, je développerai le dernier point de vue, celui de villes considérées comme des entités collectives en interaction.

\section{Des villes en système aux agents-villes}

L'idée d'appréhender les villes non en entités isolées mais les unes par rapport aux autres, est ancienne. Déjà en 1841, Raynaud évoque l'idée d' " un système général des villes ", en décrivant l'organisation hiérarchique des villes et l'imbrication de leurs différents niveaux de fonction (texte longtemps ignoré et redécouvert ${ }^{9}$ ). Et, en 1895, Élisée Reclus évoque la régularité de la configuration spatiale des villes en fonction de leur taille, avec la plus grande au centre du pays et « les villes secondaires se seraient reparties à des intervalles égaux sur le pourtour, espacées rythmi-

9 Marie-Claire Robic, "Cent ans avant Christaller... une théorie des lieux centraux ", L'Espace géographique, n 1, 1982, p. 5-12. 
quement, et chacune d'entre elles aurait eu son cortège planétaire de villes inférieures, ayant chacune son cortège de villages ${ }^{10} "$. Une formalisation plus précise, associée à une recherche d'explication fondée sur des principes d'optimisation de l'accès à des biens et à des services de niveaux variés, donnera lieu à la théorie des places centrales ${ }^{11}$. Celle-ci rend compte de la régularité des espacements entre les villes en fonction de leur niveau hiérarchique, niveau directement corrélé avec leur niveau de fonction économique. Un point de vue plus statistique, mais aussi plus universel (dans le sens où il s'applique à des objets de nature très différente, biologiques comme sociaux), est donné par la loi rang-taille de George Zipf ${ }^{12}$, exprimant la régularité à travers le temps et l'espace de la relation entre les tailles des villes et leur rang dans la hiérarchie urbaine. Pour Brian Berry ${ }^{13}$, cette régularité traduit la complexité de l'espace économique que représente la ville et la diversité des facteurs qui y interagissent. Dans son article "Cities as Systems in Systems of Cities " focalisant sur le concept de système, il préconise déjà le développement de "simulateurs urbains » qui permettent de tester les théories urbaines.

Appliquant les concepts d'Ilya Prigogine aux sciences urbaines et régionales, Peter Allen considère les villes comme des systèmes auto-organisés $^{14}$, introduit la notion de bifurcation, et propose le formalisme des systèmes d'équations différentielles pour simuler la dynamique d'un système de lieux en interaction

10 Elisée Reclus, "The Evolution of Cities », The Contemporary Review, vol. 67, n 2 , 1895 , p. 246-264.

11 Walter Christaller, «Die zentralen Örte in Süddeutschland: eine ökonomischgeographische Untersuchung über die gesetz Massigkeit der Verbreitung und Entwicklung der Siedlungen mit städitschen Funktionen », Jena, Fischer Verlag, 1933.

12 George K. Zipf, Human Behavior and the Principles of Least Effort, Cambridge (MA), Addinson-Wesley Press, 1949.

13 Brian J. L. Berry, "Cities as Systems within Systems of Cities ", Papers of the Regional Science Association, vol. 13, n 1, 1964, p. 146-163.

14 Peter Allen et Michèle Sanglier, «A Dynamic Model of Growth in a Central Place System ", Geographical Analysis, 11, 1979, p. 256-272; Peter Allen, Cities and Regions as Self-Organizing Systems; Models of Complexity, Amsterdam, Gordon and Breach Science Publishers, 1997. 
(quartiers d'une ville, villes d'une région, etc.). Ces équations décrivent l'évolution des activités et des populations de chaque lieu, en fonction des caractéristiques propres de ce lieu (économies d'agglomération et effets de saturation pris en compte à travers une fonction de la population et des emplois) et de ses possibilités d'interaction avec les autres (exprimées par la distance entre ce lieu et chacun des autres et par les complémentarités fonctionnelles existant entre eux). L'ensemble de ces termes est réuni dans une équation globale de type logistique, le facteur limitant étant évolutif. De ce fait, et du fait du caractère non linéaire des relations exprimées, ce modèle n'a pas de solution analytique et il a été exploré par simulations dans le cadre de plusieurs applications ${ }^{15}$.

Un autre champ utilisant une approche systémique est celui de la synergétique ${ }^{16}$. Il s'agit également de modéliser l'autoorganisation de systèmes complexes loin de l'équilibre. D’un point de vue théorique, cette approche lie les niveaux macro et micro, l'évolution au niveau macro étant appréhendée comme la résultante des changements au niveau individuel. Le passage formel d'un niveau à l'autre implique, en revanche, des hypothèses simplificatrices fortes comme l'homogénéité des individus en matière de choix, et leur indépendance. En conséquence, les utilités des individus correspondent simplement aux attractivités des villes, ce qui revient à supposer un parfait consensus entre les préférences individuelles. Du point de vue thématique, les mécanismes mobilisés pour rendre compte des croissances différenciées entre les villes sont donc également constitutives du niveau méso-géographique dans cette approche ${ }^{17}$. Par ailleurs, ce modèle permet de simuler l'évolution d'un système de villes avec

15 Peter Allen et Michèle Sanglier, op. cit.; Denise Pumain, Lena Sanders et Thérèse Saint-Julien, Villes et auto-organisation, Paris, Economica, 1989.

16 Herman Haken, Advanced Synergetics, Instability Hierarchies on Self-Organizing Systems and Devices, Berlin, Springer Series in Synergetics, vol. 20, 1983; Wolfgang Weidlich et Günter Haag, Concepts and Models of a Quantitative Sociology. The Dynamics of Interacting Populations, Berlin, Springer Series in Synergetics, vol. 14, 1983.

17 Günter Haag et al., "Interurban Migration and the Dynamics of a System of Cities ", Environement and Planning, vol. 24, 1992, p. 181-198. 
une conception plus large de la distance entre les villes que la seule distance physique, en intégrant ses aspects économique et culturel $^{18}$.

Des approches systémiques, s'appuyant sur les concepts d'autoorganisation, de structuration loin de l'équilibre, de bifurcation, étaient ainsi utilisées en modélisation urbaine avant l'essor des sciences de la complexité en sciences sociales et la diffusion à partir des années 1990 d'autres formalismes que les équations différentielles, celui notamment des systèmes multi-agents (SMA) et des automates cellulaires (AC). Dans les modèles utilisant les équations différentielles, il s'agit de lier par une fonction des effets opérant à un même niveau : le changement dans une ville est fonction de l'état de la variable modélisée au temps $t$, de l'état des variables influant sur elle, et de l'effet de ses interactions avec les autres villes appréhendé par sa distance à elles. Avec les systèmes multi-agents, on peut également formaliser ce type de règles liant les états de différentes variables sous la formes de lois, mais il est aussi possible, et c'est là que réside leur intérêt, de rendre compte de mécanismes "générateurs" ". On met alors l'accent sur les processus qui engendrent la configuration, la structure à laquelle on s'intéresse. La formalisation des interactions surtout en est considérablement enrichie. Dans un modèle classique fondé sur les équations différentielles, l'interaction entre deux villes est formalisée à travers une fonction liant les caractéristiques de l'une, les caractéristiques de l'autre, et la distance les séparant, ces trois éléments étant supposés expliquer l'intensité des échanges entre les deux villes (suivant les principes gravitaires notamment). Dans un SMA, la formalisation des interactions repose sur les échanges de messages entre les agents-villes, chacun d'entre eux entraînant une réaction (action-réaction). Il en émerge généralement un changement à un niveau d'observation

18 Günter Haag, Dynamic Decision Theory: Applications to Urban and Regional Topics, Dordrecht, Kluwer Acad. Pub., 1989; Lena Sanders, Systèmes de villes et synergétique, Paris, Anthropos, 1992.

19 Joshua M. Epstein, op. cit.; Gianluca Manzo, " Progrès et urgence de la modélisation en sociologie. Du concept de "modèle générateur" et de sa mise en œuvre ", L'Année sociologique, vol. 57, n 1, 2007, p. 13-61. 
supérieur, celui du système de villes et de sa structure. D’une logique d'effet et de co-évolution, on est passé à une logique de genèse et d'émergence. L'autonomie de l'agent informatique dans la gestion de ses interactions constitue un atout pour formaliser les interactions interurbaines et traduire les phénomènes d'autoorganisation qui y sont associés.

Le premier modèle mis au point suivant ce principe est le modèle SimPop, développé au sein du laboratoire Géographiecités dans les années 1990 dans le cadre d'une collaboration entre géographes et informaticiens spécialistes d'intelligence artificielle (LIP 6) ${ }^{20}$. Partant d'une grille théorique représentant une répartition à peu près régulière d'une population dont la seule activité est agricole, le modèle simule l'émergence progressive d'un système de villes hiérarchisé aux fonctions diversifiées (figure 2). La pente de la droite rang-taille ${ }^{21}$ permet de suivre le rythme du processus de hiérarchisation (figure 2c). La structure hiérarchique, observable au niveau du système de peuplement, émerge des interactions entre les entités spatiales élémentaires. Le modèle EuroSim, développé à partir d'une nouvelle plate-forme plus performante d'un point de vue technique, obéit aux mêmes principes généraux quant au rôle générateur des interactions ${ }^{22}$. Il correspond, en revanche, à une autre situation d'un point de vue théorique. Il s'agit de simuler l'évolution du système urbain européen durant un siècle, de 1950 à 2050, et de tester différents scénarios sur les effets, d'une part, de l'ouverture / la fermeture à l'immigration extra-européenne et, d'autre part, de l'existence /

20 Stéphane Bura et al., " Multi-Agents System and the Dynamics of a Settlement System ", Geographical Analysis, vol. 28, n² 2, 1996, p. 161-178; Lena Sanders et al., "SIMPOP: A Multi-Agents System for the Study of Urbanism ", Environment and Planning B, vol. 24, 1997, p. 287-305.

21 Si on représente les villes d'un pays en fonction de leur nombre d'habitants et de leur rang sur un schéma bilogarithmique, on obtient très régulièrement une courbe se rapprochant d'une droite de pente 1 (voir Michael Batty, 2001, et Denise Pumain, 2000).

22 Denise Pumain et al., "The Future of Urban Systems: Exploratory Models ", dans David Lane et al. (dir.), Complexity Perspectives on Innovation and Social Change, Springer, Methodos Series, 2009, p. 331-360; Lena Sanders et al., "Intelligence artificielle et agents collectifs : le modèle EUROSIM ", Cybergeo, n 392, 2006, p. 1-14, http://www.cybergeo.eu. 
l'absence de barrières économiques à l'intérieur de l'Europe. On part donc d'une situation initiale déjà fortement structurée, l'Europe urbaine de 1950. La plupart des applications de SMA partent de situations initiales aléatoires ou régulières (c'est le cas de SimPop) et la simulation permet justement de suivre la genèse d'une structure (spatiale et hiérarchique dans le cas de SimPop). Se pose alors la question de ce qui peut émerger lorsque la situation initiale est déjà fortement structurée. Il s'agit, dans ce cas, d'identifier les mécanismes nécessaires au maintien de la structure en place et ceux qui conduisent au contraire à un changement (passage d'une organisation hiérarchique très dominée par une ville à une organisation plus polycentrique par exemple).

Figure 2

Simulation de l'émergence d'un système de villes hiérarchisé avec le modèle SimPop : (a) situation initiale (année 0); (b) situation finale (année 2000); (c) Représentation rang-taille des villes à différentes périodes, de 0 à 2000
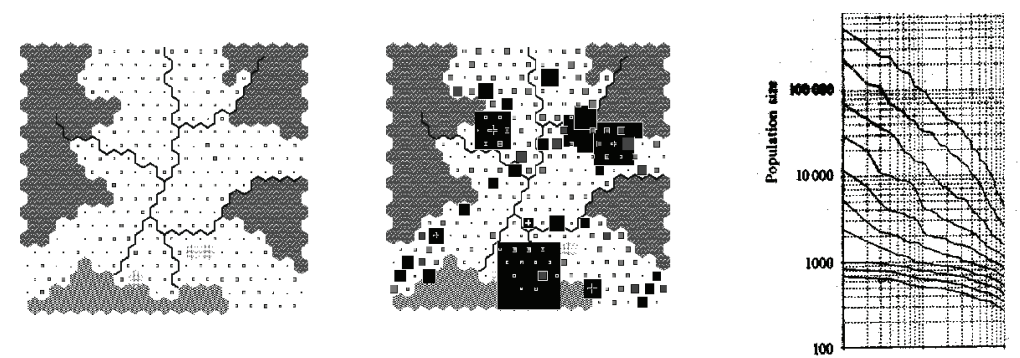

Source : Lena Sanders et al., 1997

Le modèle EuroSim permet, de plus, d'explorer l'effet de contexte. En 1950, les systèmes urbains nationaux sont déjà solidement inscrits dans l'espace et ils définissent donc le " contexte " dans lequel les villes vont évoluer. Comme l'illustre la figure 3, le réseau urbain local de Bruxelles induit d'autres potentiels d'interactions de proximité que celui de Madrid. Les contextes jouent un rôle fondamental dans la dynamique des villes, mais, dans la plupart des modèles, on l'élimine afin de se concentrer uniquement sur les caractères les plus universaux au 
détriment d'un effet de contexte tout aussi universel mais dont les modalités se déclinent évidemment différemment d'une ville à l'autre. L'approche par simulation permet de développer un " laboratoire " (système urbain artificiel) où les villes sont inscrites dans une variété de contextes urbains. Les règles d'évolution sont les mêmes pour chaque agent-ville qui les adapte en fonction de l'environnement dans lequel il est plongé. Cette approche est particulièrement intéressante lorsque l'on s'intéresse aux effets de contextes qui sont inhérents aux problématiques spatiales. Les applications les plus courantes concernent la dynamique de l'espace intra-urbain qui a mobilisé de nombreux chercheurs, avec l'objectif d'étudier les conséquences des comportements individuels, notamment les interactions entre individus et entre individus et leur environnement, sur la configuration spatiale de la ville. Les mobilités surtout ont été modélisées, qu'il s'agisse des migrations résidentielles ${ }^{23}$ ou des mobilités quotidiennes suivant des calendriers d'activité et en fonction des infrastructures de transport $^{24}$. L'échelle adoptée dans ces travaux est différente de celle utilisée dans les modèles SimPop et EuroSim mais les aspects conceptuels sont proches.

23 Itzhak Benenson, Itzhak Omer et Erez Hatna, « Entity-Based Modelling of Urban Residential Dynamics: The Case of Yaffo, Tel Aviv ", Environment and Planning B: Planning and Design, 29, 2002, p. 491-512; Yichun Xie, Mikael Batty et Kang Zhao, "Simulating Emergent Urban Form Using Agent-Based Modeling: Desakota in the Suzhou-Wuxian Region in China ", Annals of the Association of American Geographers, vol. 97, 2007, p. 477-495.

24 Arnaud Banos et al., "Simulating the Swarming City: A MAS Approach", Proceedings of the $9^{\text {th }}$ International Conference on Computers in Urban Planning and Urban Management, London, June 29-30, 2005; Philippe Mathis, "Une micro simulation dynamique urbaine basée sur le programme d'activités: utilisation du graphe cellulaire pour une simulation multi-agents ", Recherche Transports Sécurité, vol. 25/102, 2009, p. 23-45. 
Figure 3

Contextes urbains locaux de Madrid et Bruxelles

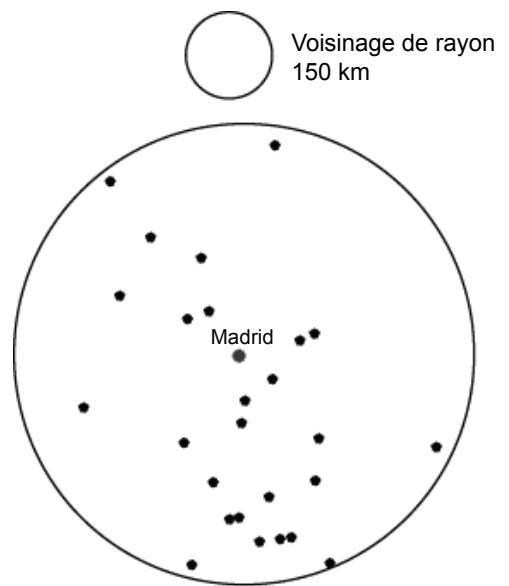

- Villes de plus de 200000 hab.

- Autres villes

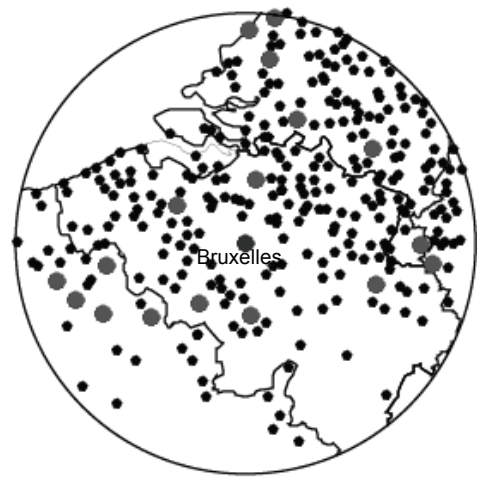

3. Dynamique des villes européennes de 1950 à 2050 : simulation et expérimentation avec le modèle EuroSim

Le modèle EuroSim se concentre sur la dynamique du haut de la hiérarchie urbaine européenne, c'est-à-dire les villes qui ont un niveau fonctionnel et/ou une spécialisation qui inscrivent leurs échanges dans les réseaux internationaux. Les prémisses du changement urbain européen sont à rechercher à ce niveau, les changements se diffusant ensuite dans le reste de la hiérarchie urbaine $^{25}$. Le choix a donc été fait dans l'application EuroSim de distinguer deux types de villes et de les formaliser avec des agents de niveaux différents :

- des agents complexes, cognitifs, pour les villes grandes (plus de 200000 habitants) et/ou spécialisées qui sont environ au nombre de 200 en 1950 et de 400 en 2050 (valeur estimée). Seules celles-ci, considérées comme motrices dans la dynamique du système urbain européen, jouent un rôle actif dans le modèle et sont capables de constituer des réseaux d'échanges;

$25 \quad$ Nadine Cattan et al., Le Système des villes européennes, $2^{\mathrm{e}}$ éd., Paris, Anthropos, coll. "Villes ", 1999. 
- des agents plus simples, réactifs, pour représenter les autres villes avec plus de 10000 habitants (au nombre de 5000 environ). Ces villes plus petites jouent un rôle plus passif, elles peuvent faire partie du réseau de villes clientes des précédentes, mais leur niveau fonctionnel n'est pas tel qu'elles puissent proposer des produits et services au niveau interurbain. En revanche, elles jouent un rôle essentiel dans la mesure où elles dessinent et spécifient le contexte dans lequel se développent les grandes villes.

Dans cette présentation relativement brève du modèle ${ }^{26}$, on se concentrera sur la formalisation des interactions dont le jeu représente la force motrice du système ${ }^{27}$. L'hypothèse qui fonde le modèle est, en effet, que la population totale et la structure de la population active évoluent en fonction de la capacité de la ville à exploiter les potentialités de son réseau d'échanges et à s'enrichir par la vente des productions associées à ses différentes spécialisations.

Chaque ville est caractérisée par ses spécialisations économiques (certaines en ont plusieurs, d'autres une seule) et sa population. À chaque pas de temps, chacune d'entre elles constitue, pour chacune de ses spécialisations, un réseau dans le cadre duquel s'effectueront ses échanges. Les villes ont simultanément deux rôles : productrices ${ }^{28}$, elles offrent leurs biens et services aux

26 Le modèle EuroSim a été développé en collaboration avec Hélène Mathian et Benoît Glisse dans le cadre du programme européen TIGRESS dirigé par Nick Winder (rapport : http://www.parisgeo.cnrs.fr/lien/tigress_2006_WP4. pdf). Ce modèle a été élaboré à partir de la plate-forme générique SIMPOP2 mise au point à l'UMR Géographie-cités dans le cadre d'une collaboration entre géographes et informaticiens (http://www.simpop.parisgeo.cnrs.fr/). Lena Sanders et Hélène Mathian, "Expérimenter sur le futur des villes européennes avec un modèle multi-agents ", $15^{\mathrm{e}}$ Journées de Rochebrune, Rencontres interdisciplinaires sur les systèmes complexes naturels et artificiels : expérimentation et systèmes complexes, ENST 2008 S 001, Paris, 2008.

La production est calculée de façon mécanique, en multipliant le nombre d'actifs dans un secteur d'activité (une spécialisation) par la productivité (avec des paramètres très généraux, différenciés par grands secteurs géographiques et par spécialisation, plus fort pour l'activité de technopole que pour l'activité industrielle, plus fort pour les activités financières que pour les spécialisations du tourisme). 
villes de leur réseaux d'échanges; consommatrices, elles achètent les biens et services correspondant à leurs demandes aux villes des réseaux d'échange desquels elles font partie. Si elles appartiennent au réseau d'échange de plusieurs villes, elles vont recevoir plusieurs propositions et devront effectuer un choix. C'est par ce biais que s'opère la concurrence entre les villes. Ces interactions sont formalisées par des échanges de messages entre les agentsvilles, ces échanges continuant jusqu'à ce qu'il n'y ait plus de demande pour l'offre proposée ou d'offre susceptible de satisfaire les demandes restantes. Il n'y a pas d'équilibre a priori entre l'offre et la demande, et il peut subsister à la fin de la période des demandes insatisfaites ou des biens et services qui n'ont pas trouvé d'acquéreur. Un pas de temps correspondant à l'année a été choisi pour la simulation : il s'agit d'une unité de temps usuelle pour appréhender le changement de taille d'une agglomération et suffisamment fine pour saisir le rythme des transformations sur une durée d'un siècle.

Les interactions opérant au sein de réseaux d'échanges, la constitution de ceux-ci représente une étape essentielle de la modélisation. Une des originalités de l'approche consiste à distinguer trois étapes :

1- la constitution du réseau potentiel d'échanges : il s'agit de sélectionner l'ensemble des villes avec lesquelles un échange pourrait potentiellement se faire étant donné la spécialisation de la ville. S'il s'agit d'activités de portée régionale, l'ensemble des villes situées à moins de $j \mathrm{~km}$ sont concernées. S'il s'agit d'activités administratives de niveau national, l'ensemble des villes d'un pays seront dans le réseau de la ville capitale. Il s'agit là de principes de proximité et territorial classiques et faciles à modéliser. En revanche, les distances et même l'appartenance nationale sont peu discriminantes pour rendre compte des échanges correspondant à des activités de haut niveau fortement spécialisées comme les finances, les technopoles, le tourisme spécialisé. L'existence de complémentarités fonctionnelles sert alors à constituer le réseau potentiel d'échanges. 
2- la constitution du réseau d'échange d'informations : une sélection est faite parmi les villes précédentes, suivant une double logique. D'une part, maintien dans le réseau des meilleures villesclientes des étapes précédentes et, d'autre part, renouvellement partiel du réseau. La sélection se fait suivant un tirage aléatoire, jusqu'à ce que la somme des demandes des villes sélectionnées dépasse $k$ fois l'offre de la ville productrice, $k$ étant un paramètre du modèle. Ainsi la taille des réseaux n’est pas fixée a priori. $K$ correspond à un critère d'arrêt et le réseau sera, assez naturellement, d'autant plus grand que les villes sélectionnées seront petites (si le tirage aléatoire favorise les grandes villes, la somme de leur demande atteindra le seuil $k$ plus rapidement que si le tirage mène à une surreprésentation des petites villes). C'est dans le réseau ainsi constitué que chaque agent-ville diffuse les messages indiquant l'importance de son offre.

3- l'émergence du réseau des transactions effectives : la plupart des villes étant simultanément pourvoyeuses de certains biens (point de vue de l'offre) et demandeuses d'autres biens, l'étape précédente donne naissance à une multitude d'échanges enchevêtrés. Chaque ville productrice a son propre réseau, et ce, pour chacune de ses spécialisations quand elle en a plusieurs. De la superposition de ces échanges simultanés résulte une compétition entre les villes. Ainsi seule une partie des échanges d'information se concrétisent par une transaction et un transfert de richesse.

L'intérêt de cette procédure de construction des réseaux d'échanges est qu'il permet de produire des réseaux évolutifs, partiellement stables et partiellement renouvelés dans leur composition. Il y a un processus d'adaptation, avec reconduction des échanges avec les villes avec lesquelles les transactions ont été fructueuses et abandon des liens infructueux. Par ailleurs, les réseaux varient d'une spécialisation à l'autre et leurs dimensions ne sont pas fixées a priori. Ces réseaux ne sont ainsi ni figés ni statiques comme l'implique le plus souvent une approche statistique ou par équations différentielles.

À chaque fin de période, un bilan économique des échanges est fait au niveau de l'agent-ville. La croissance de chaque ville 
dépend de ce bilan par l'intermédiaire de deux effets :

- un effet direct, engendré par le processus d'interaction : une demande insatisfaite est convertie en un nombre d'actifs dans la spécialisation concernée, ce nombre luimême converti en une quantité de population; au contraire, s'il reste des invendus, la population active du secteur associé sera diminuée;

- un effet indirect par l'introduction d'un mécanisme d'entraînement : le succès des transactions est supposé entraîner une plus forte attractivité de la ville, ce qui est formalisé par une augmentation du taux de croissance.

Tous les agents-villes ont les mêmes règles de fonctionnement : seuls les principes fondant la constitution des réseaux potentiels diffèrent suivant les spécialisations (principe de proximité pour les fonctions régionales, principe sélectif pour les fonctions de finance, principe de niveau de richesse pour la spécialisation touristique, etc.). Les objectifs de vendre tout ce qui est produit et d'acheter selon les besoins suivent des principes de rationalité. En revanche, le système est auto-organisé dans le sens où il n'y a pas de finalité de croissance, pas de procédure d'optimisation ni de recherche d'équilibre. Les échanges, l'ordre des messages, la diversité des propositions, suivent un processus auto-organisé dont on ne peut connaître l'issue a priori. Le système est adaptatif dans la mesure où chaque ville maintient ou modifie son réseau d'échanges en fonction des transactions qui ont abouti aux périodes précédentes.

Le modèle a été appliqué pour simuler l'évolution des villes européennes de 1950 à 2050. La période 1950-2000 a été utilisée pour calibrer les principaux paramètres du modèle. Le calibrage a visé le niveau macro-géographique, c’est-à-dire la capacité du modèle à reproduire correctement l'évolution de la population européenne totale, son organisation rang-taille, et à donner des trajectoires correctes pour les plus grandes villes du système urbain. Il ne vise pas, en revanche, à reproduire parfaitement l'ensemble des trajectoires telles qu'elles peuvent être observées au niveau méso-géographique. Il a été accompagné d'une analyse 
de sensibilité et d'une analyse de cohérence, plus importantes pour valider le modèle et pour pouvoir l'utiliser comme un laboratoire d'expérimentation pour tester différents scénarios que sa capacité à reproduire exactement la trajectoire précise de chaque ville. Un autre critère considéré comme essentiel dans la validation est la capacité du modèle à produire des comportements (ici les trajectoires) variés. L'ambition est que le modèle produise à la fois de la diversité en matière de comportements et de la diversité en matière de réponses à des scénarios différents.

Les scénarios concernent, d'une part, le degré d'ouverture des frontières européennes vis-à-vis de l'immigration venant de l'extérieur de l'Europe et, d'autre part, le degré d'ouverture / de fermeture entre les blocs de l'Est et de l'Ouest de l'Europe. Le croisement de ces hypothèses aboutit à quatre scénarios extrêmes :

- une Europe fermée vis-à-vis de l'extérieur, en déclin démographique $^{29}$, et fermée également pour ce qui concerne les échanges entre l'Est et l'Ouest. Dans ce scénario, aucun échange économique ne peut se faire entre deux villes si elles n'appartiennent pas au même bloc;

- une Europe fermée vis-à-vis de l'extérieur, mais ouverte en ce qui concerne les échanges économiques entre les blocs Est et Ouest;

- une Europe ouverte sur l'extérieur, en croissance démographique, mais sans échanges entre villes de l'Est et villes de l'Ouest;

- une Europe en croissance démographique et aux échanges économiques libres de toute contrainte.

Plusieurs simulations ont été effectuées pour chacun de ces scénarios pour la période 1950-2050. Les données simulées sur les échanges entre les villes et sur l'évolution des populations ont été stockées pour chaque date, permettant de produire et d'analyser des trajectoires au niveau de chaque ville et de

29 Les scénarios ont été construits à partir de deux hypothèses extrêmes de l'International Institute for Applied Systems Analysis (IIASA: www.iiasa.ac. at) sur les dynamiques démographiques européennes à l'horizon 2050. 
construire des indicateurs macro-géographiques sur les états successifs du système urbain européen. Il apparaît que les trajectoires simulées par le modèle sont globalement variées et que les villes répondent également de manière différente aux changements introduits par les divers scénarios. Afin d'illustrer cette capacité du modèle à reproduire de la diversité, on commentera simplement deux exemples de résultats issus des simulations, en mettant en exergue deux comportements opposés pour chacun d'entre eux.

Les trajectoires des villes artificielles de Londres et de Glasgow (figure 4) : si des différences notables apparaissent dans les deux cas entre les scénarios optimiste et pessimiste d'un point de vue démographique, ces deux villes répondent de façon différente à l'existence d'une fermeture dans les échanges économiques entre les blocs Est et Ouest. Ainsi, Londres n'est pas influencée par l'existence de contraintes sur les échanges au contraire de Glasgow. De façon plus générale, les résultats des simulations montrent que les villes possédant plusieurs spécialisations résistent mieux aux scénarios de "fermeture » que les mono-spécialisées. Le modèle reproduit ainsi un résultat qui correspond à l'observation empirique, la plus grande résilience des villes au profil économique diversifié, sans qu'une telle règle ait été explicitement introduite. 
Figure 4

Les trajectoires de population de 1950 à 2050 pour quatre scénarios pour : (a) Londres; (b) Glasgow

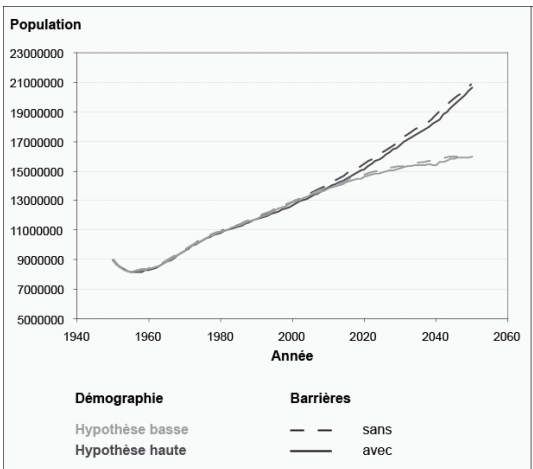

(a)

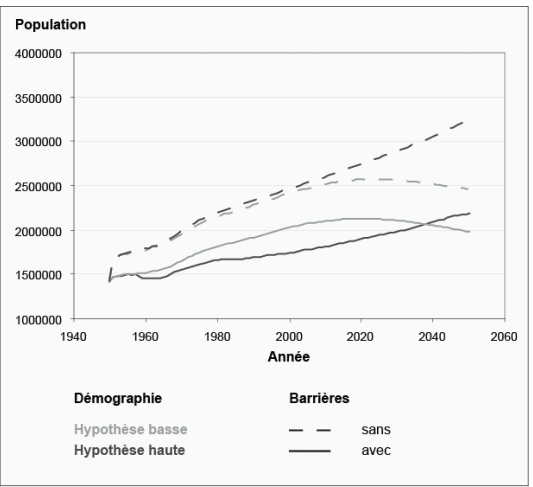

(b)

Source : Lena Sanders et Hélène Mathian, 2008

La taille des réseaux d'échanges des villes artificielles de Londres et de Budapest (figure 5): pour la première, l'absence de barrières a pour effet une extension du réseau d'échanges, résultat conforme à l'intuition, alors que pour la seconde cette absence conduit au contraire à une diminution, ce qui est $a$ priori contre-intuitif. Dans un contexte d'ouverture, la première étend son réseau d'échanges lié à sa spécialisation de technopole, alors que la seconde enregistre une diminution radicale du nombre de villes constituant son réseau d'échanges. L'absence de barrières nuit ainsi à Budapest qui souffre alors d'une concurrence plus forte pour sa spécialisation technopole. Elle perd progressivement ses villes clientes et le réseau associé à cette spécialisation va même quasiment disparaître. Néanmoins, la trajectoire démographique de la ville n'est pas affectée. Cette résilience est due à la diversité de ses spécialisations et au mécanisme de transfert des actifs d'un secteur dont la performance est en baisse vers d'autres activités. Aucune règle introduite dans le modèle ne pouvait faire attendre a priori cette différence de comportement entre Budapest et Londres, elle résulte du jeu des interactions entre les sous-ensembles de villes. Et cette différence qui émerge des interactions 
croisées des villes au sein de leurs réseaux respectifs est en accord avec la théorie urbaine ${ }^{30}$.

Figure 5

Évolution de la taille des réseaux d'échanges pour une série de simulations : (a) Londres; (b) Budapest

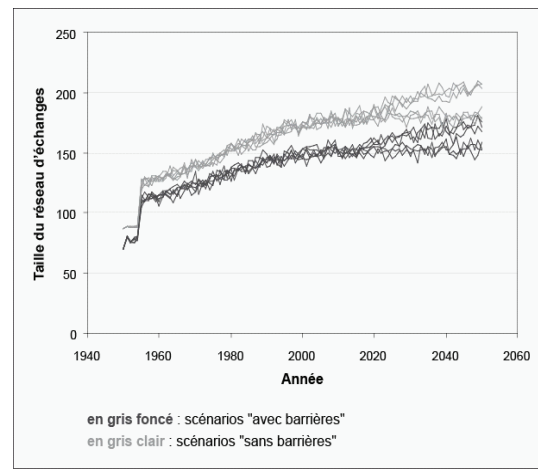

(a)

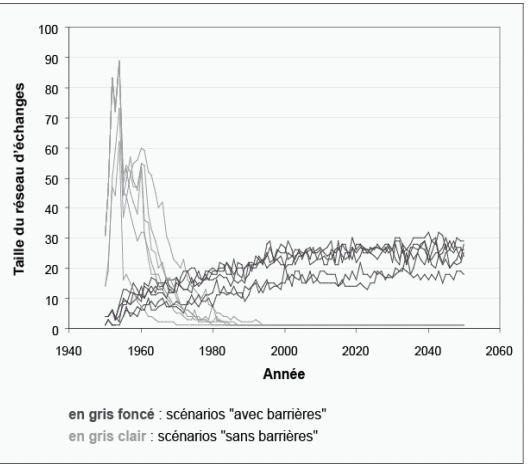

(b)

Source : Lena Sanders et Hélène Mathian, 2008

\section{Perspectives : vers une approche combinant principe d'auto-organisation et action des acteurs urbains}

Dans la discussion sur le niveau de modélisation deux types d'acteurs sont considérés : 1) ceux que l'on a qualifiés d'individus et qui représentent les habitants ordinaires dont les préférences et les choix peuvent avoir un effet sur le niveau méso-géographique à travers un processus bottom-up (ce niveau n'a pas été appréhendé dans le modèle EuroSim); 2) les acteurs urbains, en tant que macro acteurs (maires, élus, chefs d'entreprise) dont les décisions concernent directement le niveau de la ville. Ces derniers relèvent du même niveau méso-géographique que les règles introduites dans le modèle EuroSim, mais réfèrent à des modes d'explication qui paraissent opposés de plusieurs points de vue : opposition entre régularités et spécificités, lois et actions, auto-organisation

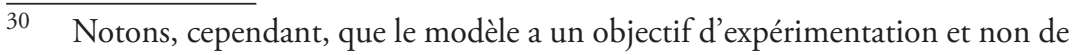
prévision, et aucune conclusion ne doit être tirée sur ce que seront Londres et Budapest en l'an 2050. 
et fixation d'un objectif. D'un point de vue thématique, il est plus intéressant de réfléchir à la façon de prendre en compte les complémentarités de ces approches que de débattre sur les oppositions dont elles sont porteuses. On peut ainsi proposer l'articulation suivante : les règles générales et les mécanismes d'auto-organisation définissent les bornes des futurs possibles des villes en fonction de leur population, de leur spécialisation et du contexte spatial dans lequel elles sont situées (c'est-à-dire la proximité des autres villes selon leur taille, la densité du réseau urbain local dans lequel elles sont insérées); et les décisions des acteurs contribuent à orienter une trajectoire donnée vers l'un ou l'autre bord de l'intervalle ainsi défini. L'hypothèse est ainsi que les stratégies mises au point par les acteurs d'une ville influencent l'évolution de la ville, mais seulement à l'intérieur du champ de possibilité défini par un modèle systémique du type de EuroSim.

Ce modèle n'a pas pour objectif de prévoir l'évolution de la population de chaque ville de manière précise. Pour ce faire, il apparaît que les meilleures prévisions à court terme sont celles correspondant au prolongement des tendances récentes, et ne référant à aucun cadre explicatif. Il s'agit plutôt d'aider à réfléchir, à anticiper, l'impact de certaines décisions ou de certains événements, sur le devenir des villes sur le temps long. La mise en évidence de résultats contre-intuitifs, car issus de mécanismes non linéaires et indirects, peut en particulier constituer une aide à la décision. La création de nouveaux emplois dans un secteur donné n’aura pas les mêmes conséquences suivant la spécialisation de la ville, les caractéristiques de son environnement et sa place dans les réseaux européens d'échange. La donnée (par le modèle) d'un ensemble de trajectoires possibles donne un point de départ à la réflexion du décideur pour définir ses objectifs de croissance pour la ville. En ce sens, de tels modèles représentent des aides à la réflexion de l'acteur politique.

Par ailleurs, il est possible d'approfondir plus formellement l'analyse des effets réciproques des principes auto-organisateurs et des stratégies d'acteurs sur l'évolution des villes. La plate-forme 
SIMPOP2 (voir note 3) permet d'introduire des comportements d'acteurs à côté des règles générales qui ont été évoquées précédemment. Le système urbain artificiel ainsi constitué devrait permettre de tester les effets de comportements spécifiques, c'est-à-dire d'impulsions données à une ou quelques villes seulement, à court et à long terme. On pourrait ainsi introduire des décisions d'ordre varié comme celle d'élargir le réseau d'échanges de la ville pour une activité donnée, ou transférer des emplois d'un secteur à un autre (pour une ville qui a plusieurs spécialisations), afin d'en tester les effets à long terme sur les villes initiatrices et sur les autres. On peut aussi introduire des stratégies plus complexes comme une imitation des villes possédant la même spécialisation et réussissant mieux, en empiétant, par exemple, sur leurs réseaux de clients. La répétition de telles expérimentations permettrait de cerner les poids respectif des règles générales de fonctionnement du système de villes et des actions spécifiques menées au niveau local, et de mettre ainsi en évidence quelles sont les marges de manœuvre des acteurs politiques.

\section{Bibliographie}

Allen, Peter, Cities and Regions as Self-Organizing Systems; Models of Complexity, Amsterdam, Gordon and Breach Science Publishers, 1997.

Allen, Peter et Michèle Sanglier, "A Dynamic Model of Growth in a Central Place System ", Geographical Analysis, 11, 1979, p. 256-272.

Amblard, Frédéric et Denis Phan, Modélisation et simulation multi-agents : applications pour les sciences de l'homme et de la société, Paris, HermesLavoisier, 2006.

Banos, Arnaud, Sonia Chardonnel, Christophe Lang, Nicolas Marilleau et Thomas Thévenin, "Simulating the Swarming city: A MAS Approach ", Proceedings of the $9^{\text {th }}$ International Conference on Computers in Urban Planning and Urban Management, London, June 29-30, 2005. 
Batty, Michael, "Polynucleated Urban Landscapes ", Urban Studies, vol. 38, no 4, 2001, p. 635-655.

Benenson, Itzhak, Itzhak Omer et Erez Hatna, « Entity-Based Modelling of Urban Residential Dynamics: The Case of Yaffo, Tel Aviv ", Environment and Planning B: Planning and Design, 29, 2002, p. 491512.

Berry, Brian J. L., "Cities as Systems Within Systems of Cities ", Papers of the Regional Science Association, vol. 13, nº 1, 1964, p. 146-163.

Bretagnolle, Anne, Villes et réseaux de transport, des interactions dans la longue durée (France, Europe, États-Unis), Habilitation à Diriger des Recherches, Université Paris 1, 2009.

Bura, Stéphane, France Guèrin-Pace, Hélène Mathian, Denise Pumain et Lena Sanders, «Multi-Agents System and the Dynamics of a Settlement System ", Geographical Analysis, vol. 28, n² 2, 1996, p. 161-178.

Cattan, Nadine, Denise Pumain, Céline Rozenblat et Thérèse Saint-Julien, Le Système des villes européennes, $2^{e}$ éd., Paris, Anthropos, coll. "Villes ", 1999.

Christaller, Walter, « Die zentralen Örte in Süddeutschland: eine ökonomisch-geographische Untersuchung über die gesetz Massigkeit der Verbreitung und Entwicklung der Siedlungen mit städitschen Funktionen ", Jena, Fischer Verlag, 1933.

Clarke, Graham (dir.), Microsimulation for Urban and Regional Policy Analysis, London, Pion, 1996.

Epstein, Joshua M., Generative Social Science: Studies in Agent-Based Computational Modeling, Princeton, Princeton University Press, 2006.

Favory, François et al., "Sélection géographique, déterminisme et hasard ", dans Archaeomedes (collectif de direction), Des oppida aux métropoles, Paris, Anthropos, coll. "Villes », 1998, p. 151-248.

Guerois, Marianne et Fabien Paulus, "Commune centre, agglomération, aire urbaine : quelle pertinence pour l'étude des villes? ", Cybergeo, $\mathrm{n}^{\circ} 212,2002$, p. 1-18.

Haag, Günter, Dynamic Decision Theory: Applications to Urban and Regional Topics, Dordrecht, Kluwer Acad. Pub., 1989.

Haag, Günter et al., «Interurban Migration and the Dynamics of a System of Cities ", Environement and Planning, vol. 24, 1992, p. 181-198.

Haken, Herman, Advanced Synergetics, Instability Hierarchies on SelfOrganizing Systems and Devices, Berlin, Springer Series in Synergetics, vol. $20,1983$. 
Holm, Einar, Urban Lindgren et Gunnar Malmberg, "Dynamic Microsimulation ", dans Fotheringham, Stewart et Mikael Wegener (dir.), Spatial Models and GIS: New Potential and New Models, GISDATA 7, Taylor \& Francis, 2000, p. 143-165.

Kohler, Timothy A. et George J. Gumerman (dir.), Dynamics in Human and Primate Societies. Agent-Based Modeling of Social and Spatial Processes, Oxford, Oxford University Press, 2000.

Le Gales, Patrick, Le retour des villes européennes. Sociétés urbaines, mondialisation, gouvernement et gouvernance, Paris, Presses de Sciences Po, 2003.

Manzo, Gianluca, " Progrès et urgence de la modélisation en sociologie. Du concept de "modèle générateur" et de sa mise en œuvre ", L'Année sociologique, vol. $57, \mathrm{n}^{\circ} 1,2007$, p. 13-61.

Mathis, Philippe, «Une micro simulation dynamique urbaine basée sur le programme d'activités: utilisation du graphe cellulaire pour une simulation multi-agents ", Recherche Transports Sécurité, vol. 25/102, 2009, p. 23-45.

Portugali, Juval, "Toward a Cognitive Approach to Urban Dynamics ", Environment and Planning B: Planning and Design, vol. 31, $\mathrm{n}^{\circ}$ 4, 2004, p. 589-613.

Pumain, Denise, "Settlement Systems in the Evolution ", Geografiska Annaler, 82B, 2, 2000, p. 73-87.

Pumain, Denise, Lena Sanders et Thérèse Saint-Julien, Villes et autoorganisation, Paris, Economica, 1989.

Pumain, Denise, Lena Sanders, Anne Bretagnolle, Benoît Glisse et Hélène Mathian, "The Future of Urban Systems: Exploratory Models ", dans David Lane, Sander van der Leeuw, Geoffray West et Denise Pumain (dir.), Complexity Perspectives on Innovation and Social Change, Springer, Methodos Series, 2009, p. 331-360.

Robic, Marie-Claire, "Cent ans avant Christaller... une théorie des lieux centraux ", L'Espace géographique, n 1, 1982, p. 5-12.

Reclus, Elisée, "The Evolution of Cities ", The Contemporary Review, vol. 67, $\mathrm{n}^{\circ} 2,1895$, p. 246-264.

Sanders, Lena, Systèmes de villes et synergétique, Paris, Anthropos, 1992.

Sanders, Lena et Hélène Mathian, "Expérimenter sur le futur des villes

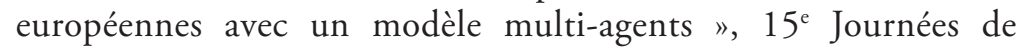
Rochebrune, Rencontres interdisciplinaires sur les systèmes complexes naturels et artificiels: expérimentation et systèmes complexes, ENST 2008 S 001, Paris, 2008. 
Sanders, Lena, Jean-Marc Favaro, Hélène Mathian, Denise Pumain et Benoît Glisse, «Intelligence artificielle et agents collectifs : le modèle EUROSIM », Cybergeo, n 392, 2006, p. 1-14, http://www.cybergeo. $\mathrm{eu} /$.

Sanders, Lena et al., "SIMPOP: A Multi-Agents System for the Study of Urbanism ", Environment and Planning B, vol. 24, 1997, p. 287-305.

Waddell, Paul, « UrbanSim: Modeling Urban Development for Land Use, Transportation and Environmental Planning ", Journal of the American Planning Association, vol. 68, n 3, 2002, p. 297-314.

Wagner, Peter et Michael Wegener, "Urban Land Use, Transport and Environment Models. Experiences with an Integrated Microscopic Approach ", disP 170, 3, 2007, p. 45-56.

Weidlich, Wolfgang et Günter Haag, Concepts and Models of a Quantitative Sociology. The Dynamics of Interacting Populations, Berlin, Springer Series in Synergetics, vol. 14, 1983.

Xie, Yichun, Mikael Batty et Kang Zhao, "Simulating Emergent Urban Form Using Agent-Based Modeling: Desakota in the Suzhou-Wuxian Region in China ", Annals of the Association of American Geographers, vol. 97, 2007, p. 477-495.

Zipf, George K., Human Behavior and the Principles of Least Effort, Cambridge (MA), Addinson-Wesley Press, 1949. 